\title{
Neuroplasticity vs. cognitive prediction models in acute ischaemic stroke: limitations must be remembered
}

\author{
Ingrid Xiomara Tibocha-Gordon ${ }^{1}$, Vanessa Morales-Ospina ${ }^{2}$, \\ Yudex Abraham Vergara-Jacome ${ }^{1}$, Ivan David Lozada-Martínez ${ }^{3,4}$ \\ ${ }^{1}$ School of Medicine, University Corporation Rafael Nuñez, Cartagena, Colombia \\ ${ }^{2}$ School of Medicine, Pontifical Bolivarian University, Bogotá, Colombia \\ ${ }^{3}$ Colombian Clinical Research Group in Neurocritical Care, University of Cartagena, Cartagena, Colombia \\ ${ }^{4}$ Latin American Council of Neurocritical Care, Cartagena, Colombia
}

Key words: ischaemic stroke, neuronal plasticity, stroke rehabilitation, cerebrovascular disorders, prognosis

(Neurol Neurochir Pol 2022; 56 (1): 101-102)

\section{To the Editors,}

We read with great interest the article by Esmael et al. [1] entitled 'Prevalence of cognitive impairment in acute ischaemic stroke and use of Alberta Stroke Programme Early CT Score (ASPECTS) for early prediction of post-stroke cognitive impairment', where the authors evaluated the predictive value of ASPECTS on post-stroke cognitive improvement, stating that it should be a predictive biomarker due to its correlation with improved cognition at a three-month follow-up [1].

We thank Esmael et al. [1] for providing such valuable evidence. However, we would like to make some comments on the role of neuroplasticity and neurorehabilitation as determinants of the post-stroke recovery process, regarding what we see as clear limitations to the use of predictive models based on algorithms.

Neuroplasticity is the capacity of the brain to reorganise and reconstruct neuronal connections secondary to modifications in its environment [2]. This process occurs thanks to a great variety of gene expression in response to the presence of external stimuli (epigenetic) or brain functional reserve (genetic self-factors) [2,3].

This complex concept has been the subject of extensive research in recent years, which has resulted in finding that physical exercise [4], environment, cognitive stimulation [5], social interaction and previous pathological conditions all substantially influence the final outcome of this process [3-5]. These findings suggest that the process of neuroplasticity is the result of multiple factors, and that its course is personalised, and therefore highly unpredictable.

Although there are many pathways to generate an effective neuroplasticity process $[2,3,5]$, such as neurogenesis, angiogenesis, gliogenesis, axonal sprouting and growth or synaptic plasticity (which result from the activation of molecular mechanisms such as DNA methylation, chromatin modifications, microRNAs activation, histone modification, among others), there are factors conditioned to aspects such as compensatory adaptation which is reinforced depending on the brain territory affected by stroke or any other vascular or non-vascular neurological disorder $[2,3,5]$. At this point, there is a need to start early the neurorehabilitation process in a patient with neurological sequelae following a neurological disorder, since the activities aimed at stimulating the affected brain territory, are those that will impact conditionally on the functional capacity and outcome of the patient [5].

Based on the above evidence, there is an urgent need to create specialised neurorehabilitation centres in low- and middle-income countries where there is a very high burden of disease due to cerebrovascular disorders, and where there are many barriers within the healthcare system [6], with the aim of improving functional capacity and quality of life, as well as reducing morbidity and mortality.

Address for correspondence: Ivan David Lozada-Martinez, Colombian Clinical Research Group in Neurocritical Care, University of Cartagena, Cartagena, Colombia; e-mail: ilozadam@unicartagena.edu.co

Submitted: 26.05.2021 Accepted: 22.06.2021 Early publication date: 3.08.2021

This article is available in open access under Creative Common Attribution-Non-Commercial-No Derivatives 4.0 International (CC BY-NC-ND 4.0) license, allowing to download articles and share them with others as long as they credit the authors and the publisher, but without permission to change them in any way or use them commercially. 
Although predictive models are innovative tools that allow us to observe changes over time, they are limited by the influence of a large number of factors on neuroplasticity, so it is not possible to extrapolate or accurately determine a prognosis.

For example, one cannot expect the same recovery from a middle-aged research professional from a high-income country, who has a high degree of education and due to his occupation reads constantly, interacts with academics, and probably has the economic resources to access an adequate neurorehabilitation plan, as we can from a middle-aged worker from a low- or middle-income country, who has no education whatsoever, or from a patient who has a history of any neurological disorder, or from those who present repeated transient ischaemic strokes in the post-stroke period.

There are numerous examples of groups of patients who may present an unpredictable evolution. There are also potential limitations reported in the use of the Montreal Cognitive Assessment (MoCA) as a cognitive impairment screening tool [7], where the correspondence between individual tests and their assumed cognitive domains is not robust, reflecting at least in part a current lack of consensus as to how core cognitive constructs are defined and as to which subcomponents can be subsumed under different cognitive domains [7]. These limitations among the correlations cause the cut-off scores published in the literature to diverge, and do not allow accurate prediction of cognitive impairment [8].

In order to obtain a truly personalised predictive value, it would be necessary to use neuroimaging very often during the patient's follow-up period, which would not be cost-effective, nor in the patient's own interest given the radiation exposure in the case of CT scanning.

Therefore, we consider that it will be complex, but not impossible, to develop a predictive tool based on genetic and epigenetic characteristics of the stroke patient which takes into account the degree of brain functional reserve and the activity of its neuroplasticity.
Funding: None.

Conflicts of interest: None.

\section{References}

1. Esmael A, Elsherief M, Eltoukhy K. Prevalence of cognitive impairment in acute ischaemic stroke and use of Alberta Stroke Programme Early CT Score (ASPECTS) for early prediction of post-stroke cognitive impairment. Neurol Neurochir Pol. 2021; 55(2): 179-185, doi: 10.5603/PJNNS.a2021.0006, indexed in Pubmed: 33507530.

2. Dąbrowski J, Czajka A, Zielińska-Turek J, et al. Brain functional reserve in the context of neuroplasticity after stroke. Neural Plast. 2019; 2019: 9708905, doi: 10.1155/2019/9708905, indexed in Pubmed: 30936915.

3. Felling RJ, Song H. Epigenetic mechanisms of neuroplasticity and the implications for stroke recovery. Exp Neurol. 2015; 268: 37-45, doi: 10.1016/j.expneurol.2014.09.017, indexed in Pubmed: 25263580.

4. Xing Y, Bai Y. A review of exercise-induced neuroplasticity in ischemic stroke: pathology and mechanisms. Mol Neurobiol. 2020; 57(10): 4218-4231, doi: 10.1007/s12035-020-02021-1, indexed in Pubmed: 32691303.

5. Farokhi-Sisakht F, Farhoudi M, Sadigh-Eteghad S, et al. Cognitive rehabilitation improves ischemic stroke-induced cognitive impairment: role of growth factors. J Stroke Cerebrovasc Dis. 2019; 28(10): 104299, doi: 10.1016/j.jstrokecerebrovasdis.2019.07.015, indexed in Pubmed: 31371141.

6. World Health Organization. Global Burden of Neurological Disorders: Estimates and Projections. https://www.who.int/mental_health/neurology/chapter_2_neuro_disorders_public_h_challenges.pdf (Consulted 26 May 2021).

7. Coen RF, Robertson DA, Kenny RA, et al. Strengths and limitations of the MoCA for assessing cognitive functioning: findings from a large representative sample of Irish older adults. J Geriatr Psychiatry Neurol. 2016; 29(1): 18-24, doi: 10.1177/0891988715598236, indexed in Pubmed: 26251108.

8. Sokołowska N, Sokołowski R, Oleksy E, et al. Usefulness of the Polish versions of the Montreal Cognitive Assessment 7.2 and the Mini-Mental State Examination as screening instruments for the detection of mild neurocognitive disorder. Neurol Neurochir Pol. 2020; 54(5): 440-448, doi: 10.5603/PJNNS.a2020.0064, indexed in Pubmed: 32808669. 